\title{
Early azathioprine in acute Vogt-Koyanagi-Harada disease: a prospective 24-month follow-up study with multimodal imaging and electroretinogram
}

Marcelo Mendes Lavezzo ( $\sim$ mmlavezzo@gmail.com )

Hospital das Clínicas da Faculdade de Medicina da Universidade de São Paulo

Viviane Mayumi Sakata

Hospital de Clínicas Universidade Federal do Paraná

Fernanda Maria Silveira Souto

Hospital das Clínicas da Faculdade de Medicina da Universidade de São Paulo

Ruy Felippe Brito Gonçalves Missaka

Hospital das Clínicas da Faculdade de Medicina da Universidade de São Paulo

Priscilla Figueiredo Campos da Nobrega

Hospital das Clínicas da Faculdade de Medicina da Universidade de São Paulo

Maria Kiyoko Oyamada

Hospital das Clínicas da Faculdade de Medicina da Universidade de São Paulo

Carlos Eduardo Hirata

Hospital das Clínicas da Faculdade de Medicina da Universidade de São Paulo

Joyce Hisae Yamamoto

Hospital das Clínicas da Faculdade de Medicina da Universidade de São Paulo

\section{Research Article}

Keywords: Vogt-Koyanagi-Harada disease; immunosuppression, indocyanine green angiography, subclinical signs, electroretinogram

Posted Date: January 13th, 2022

DOI: https://doi.org/10.21203/rs.3.rs-1251714/v1

License: (c) (i) This work is licensed under a Creative Commons Attribution 4.0 International License. Read Full License 


\section{Abstract}

Background: First-line immunosuppressive therapy (IMT) associated with high-dose corticosteroid (CS) has been proposed for the treatment of acute Vogt-Koyanagi-Harada disease (VKHD) to prevent chronicity and to prevent long-term CS side effects. However, there are very few studies that systematically evaluated visual and inflammatory outcomes in acute VKHD with early IMT. This study aimed to evaluate the outcome of high-dose corticosteroids with early addition of azathioprine (AZA) in patients with acute Vogt-Koyanagi-Harada disease (VKHD) followed for 24-month with systematic multimodal and electroretinogram exams.

Methods: Prospective interventional study. Fifteen consecutive patients (30 eyes) with acute VKHD at a tertiary uveitis referral centre were followed for 24 months with systematic multimodal and full-field electroretinogram (ffERG) exams. Patients were treated with intravenous methylprednisolone followed by oral prednisone $1 \mathrm{mg} / \mathrm{kg} /$ daily (CS) with slow taper and AZA introduction within 4 months. Anterior uveitis relapse, subclinical inflammation, best-corrected visual acuity (BCVA) and ffERG parameters were analyzed.

Results: Fifteen patients ( 14 female) with a median age of 32 years were included. In the first month, 27 eyes $(90 \%)$ had BCVA $\geq 20 / 40$; at M24, all eyes $(100 \%)$ had BCVA $\geq 20 / 25$. Uveitis resolved in 28 eyes $(93.3 \%)$ and became chronic recurrent in 2 eyes $(6.7 \%)$; subclinical inflammation was still present in all eyes during the 24-month follow-up. ffERG parameters initially improved in all eyes; at M24, 23 eyes (76.7\%) had subnormal results and 20 eyes $(66.7 \%)$ had stable parameters. Eyes with very early treatment $(n=12)$ had lower indocyanine green angiography score than eyes with early treatment $(n=18)$ at M1 $(p=0.012)$, but they had similar rates of recurrence, complications and ffERG parameters.

Conclusion: Early AZA associated with high-dose corticosteroid was effective in improving BCVA and in controlling clinical inflammation. Isolate subclinical inflammation persisted in all eyes with no impact on ffERG in, at least, two thirds of these eyes, indicating that isolate subclinical inflammation may not be enough to indicate treatment increment.

\section{Introduction}

Vogt-Koyanagi-Harada disease (VKHD), a systemic T cell-mediated autoimmune disorder that targets melanocyte-specific proteins $(1,2)$, is characterized as a stromal choroiditis, with exudative retinal detachment and optic disc hyperemia/swelling, followed by anterior segment involvement (1, 3-5). It corresponds to $2.5-12.5 \%$ of uveitis in Brazilian referral centers(6-8) whereas its prevalence varies broadly, being uncommon in Europe(9) and USA(10) and more frequent in East Asia and Southeast Asia $(11,12)$. The standard treatment with early high-dose corticosteroid (CS) rapidly controls the early signs of inflammation with prompt recovery of visual acuity(13-15). However, the inflammation became chronic or recurrent, manifested as recurrent anterior uveitis or subclinical choroidal inflammation, in almost twothirds of patients $(13,16)$. Association of immunosuppressive drug therapy (IMT) reduces risk of vision 
loss as well as imposes a better control of inflammation in CS refractory/intolerant cases $(1,17)$. More recently, several studies propose early IMT associated with high-dose CS as a step towards preventing CS long-term deleterious side effects and avoiding chronicity(18-20).

In this context, multimodal imaging of posterior segment inflammatory signs, including fluorescein angiography (FA), indocyanine green angiography (ICGA) and enhanced depth imaging optical coherence tomography (EDI-OCT), brought light to subclinical choroiditis in non-acute $\operatorname{VKHD}(4,13,21)$. There are to date few systematic studies evaluating the relevance of this subclinical choroiditis on visual function outcomes and consequently on tailoring treatment.

Our previous prospective study, including 12 patients with acute onset VKHD treated with initial high-dose CS and slow tapering associated with late IMT, demonstrated an optimal control of acute inflammatory signs with VA $\geq 20 / 25$ in more than $90 \%$ of patients. Nevertheless, 6 months after disease onset and during the 24-month follow-up, anterior uveitis relapse was observed in $33.3 \%$ of patients and subclinical choroiditis was present in $91.7 \%$ of patients. Full-field electroretinogram (ffERG) parameters were stable during the follow-up in up to $71 \%$ of eyes despite the presence of subclinical choroiditis(13). Based on these previous results and on recent strong indication of early IMT in acute VKHD, the present study aimed to evaluate the outcome of high-dose corticosteroids with early addition of azathioprine (AZA) in patients with acute VKHD followed for 24-month with systematic multimodal and electroretinogram exams.

\section{Subjects And Methods}

This prospective study included 15 consecutive patients with acute onset VKHD(22) attended at Uveitis Service, Hospital das Clínicas HCFMUSP, Faculdade de Medicina, Universidade de Sao Paulo, Sao Paulo, SP, Brazil, from September/2015 to February/2018. All patients were followed for at least 24 months. Patients who had other causes of uveitis, media opacities, refractive errors $>5$ diopters, and/or systemic contraindication to IMT were excluded. The local institutional review board approved the full protocol, which follows the tenets of the declaration of Helsinki (clinical trials NCT03399175).

All patients were treated with a three-day course of intravenous methylprednisolone ( $1000 \mathrm{mg} /$ day $)$ pulse therapy, followed by a $1.0 \mathrm{mg} / \mathrm{kg} /$ daily oral prednisone with slow taper during at least 9 months (Supplemental Table 1). In case of persistent clinical inflammation, CS tapering was postponed. Oral azathioprine ( $2 \mathrm{mg} / \mathrm{kg} /$ day; maximum $3 \mathrm{mg} / \mathrm{kg} /$ day) was added up in the four first months after pulse therapy. Azathioprine was switched to mycophenolate mofetil $(2 \mathrm{~g} /$ day; maximum $3 \mathrm{~g} /$ day) due to intolerance and/or refractory disease, in spite of optimum azathioprine dosage.

\section{Multimodal analysis}

Best-corrected visual acuity (BCVA), measured with the Snellen chart, was converted to LogMAR values. Systematic clinical and multimodal imaging exams were performed at inclusion, 1, 2, 4, 6 months (M), and, thereafter, every 3 months. Multimodal imaging examinations included fundus color photography 
(Topcon TRC-50DX, Tokyo, Japan), FA, ICGA and EDI-OCT (Spectralis® HRA+OCT, Heidelberg Engineering, Germany). The ffERG recording was performed at inclusion, 30 days and at 6-month intervals thereafter. An additional ffERG recording was carried out based on the presence of subclinical signs, as described below.

Fundus findings were graded according to a previously described analytical framework(23). The development of SGF was classified as either present or absent, based on the fundus color photography. FA and ICGA reading charts were based on an angiographic scoring system for uveitis(24). For the followup, we also evaluated dark dots (DD) alone, since they are the most constant and easily recordable ICGA sign(25). The total DD score varied from 0 to 8(24). The fluctuation with worsening was defined as a change in $\mathrm{DD}$ score $\geq 0.5$ between three consecutive time points(24).

ffERG recordings were performed following the ISCEV guidelines with the RETI-port system (Roland Consult@; Electrophysiological Diagnostic Systems, Brandenburg, Germany) using ERG-jet electrodes (Universe SA, La Chaux-de-Fons, Switzerland)(26). The recording sequence was scotopic rod response, scotopic cone-rod combined response, scotopic oscillatory potentials, photopic cone response and $30-\mathrm{Hz}$ flicker cone response. The normal value limits for each specific ERG response were established at $95 \%$ confidence intervals. Amplitude of $a$ and $b$ waves of eight averaged stimulus responses for each phase were analyzed. The results were compared with a normative database, consisting of 30 healthy subjects (60 eyes) matched for age and sex. During the follow-up, value reduction $\geq 30 \%$ (confirmed in two sequential exams)(27) in any ffERG parameter in consecutive exams indicated an increase of IMT. A subnormal ffERG recording was defined as the presence of at least one parameter below the 5th percentile of healthy age, gender-matched controls. ffERG measurements stability or worsening obtained at 12 and 24 months were compared; worsening was defined as at least $31 \%$ change in any of the parameters, except for flicker, in which at least a $44 \%$ change was considered $(28,29)$ For this study implicit time was not included for the analysis(30).

After the acute inflammatory phase ( 6 months following disease onset), the disease activity was systematically evaluated based on both clinical and subclinical signs. Clinical signs included anterior chamber cells (ACC) of at least 1+ cells(31); exudative retinal detachment; choroidal neovascularization (CNV) revealed by fundus examination and/or OCT and/or FA, and macular edema confirmed on FA and/or OCT. Subclinical signs included optic disc leakage/hyperfluorescence and/or perivascular leakage on FA; DD persistence or score fluctuation with worsening on ICGA and subfoveal choroidal thickness (CT) increase (defined as an increase $\geq 30 \%$ ) on EDI-OCT.

In this prospective study protocol, the need for systemic IMT increment was based solely on the presence of clinical signs and/or worsening of ffERG exam. Isolated subclinical signs of activity did not infer a change in treatment schedule, but the patient was directed to the ffERG exam. If ffERG parameters worsened as described above, IMT was increased. The study flow chart is demonstrated in Supplemental Figure 1. Based on the predefined protocol, the term "flare" was used to describe the appearance or worsening of inflammatory signs observed at least 6 months after disease onset. 
During the 24-month follow-up period, the presence of ocular complications was recorded. CNV was diagnosed on FA and/or on OCT and was treated with an intravitreal bevacizumab injection and IMT increment(32).

The primary outcomes were clinical and subclinical flares, BCVA and ffERG results at 24 months of follow-up. The frequency of ocular complications was observed, as a secondary outcome as well as CSE, defined as a prednisone dose $\leq 10 \mathrm{mg} /$ day while maintaining clinically inactive uveitis.

\section{Statistical analysis}

For the descriptive data analysis, medians, means and standard deviations were calculated for numerical variables and absolute/relative frequencies were presented for qualitative variables (Table 1). Patients had both eyes included for analysis, therefore, generalized estimation equations (GEE) were used to evaluate the difference between repeated measurements using the appropriate correction for inter-eye dependency (Tables 2 and 3, Supplemental Table 2). GEE with Poisson's distribution and identity link function, likelihood ratio, Kruskal-Wallis test and Fisher exact test were used when comparing different intervals to start treatment. (Table 3 and Supplemental Table 2). $P$ values $\leq 0.05$ were considered significant. Data analysis and statistical tests were conducted using SPSS20.0 (SPSS Science, Chicago, IL, USA).

\section{Results}

Fifteen patients (14 women; 30 eyes) with acute VKHD were included in this study. The median age was 32 years (range: $19-46$ years). During the 24-month follow-up, four patients (26.7\%), eight patients $(53.3 \%)$ and three patients (20\%) had complete, incomplete and probable disease, respectively(22). Five patients $(33.3 \%)$ carried the HLA-DRB $1 * 0405$ allele. The median interval between onset of symptoms and treatment was 19 days (range: 8-57 days). The median interval to azathioprine introduction was 32 days (range: 0-97 days). Considering the therapeutic dose of azathioprine ( $2 \mathrm{mg} / \mathrm{kg} /$ day), it was achieved within the first four months in 13 patients (86.7\%). During the acute phase, 26 eyes (86.7\%) presented panuveitis (4 eyes had trace cells in the anterior chamber) and 29 eyes $(96.7 \%)$ revealed serous retinal detachment. Median FA scores and ICGA at baseline were 6.5 (range: 2-11) and 11.5 (range: 6-17), respectively (Table 1 ).

Acute inflammatory signs rapidly subsided in all eyes after treatment. In the first month, 27 eyes (90\%) had BCVA $\geq 20 / 40$ (logMAR $\leq 0.3$ ); at M24, all eyes $(100 \%)$ had BCVA $\geq 20 / 25$ (logMAR $\leq 0.1$ ). Subfoveal CT decreased in all eyes from the baseline (median $774 \mu \mathrm{m}$; range: $454-1167 \mu \mathrm{m}$ ) to M1 (median 529.5 $\mu \mathrm{m}$; range: $310-940 \mu \mathrm{m}$ ) and continued to decrease till the last included follow-up (M24). The median time to optic disc leakage improvement was two months (range: 0-9 months). ICGA total score at the first month decreased (median 11.5, range 6-17 at baseline to 8, range 5-13 at M1) mainly due improvement of optic disc and early stromal vessels hyperfluorescence and choroidal vasculitis (fuzzy vessels). DD scores hardly improved during the first month; at M12, they decreased in 26 eyes (86.7\%) with a median score of 5 (range: 4-8) (Table 1). 
Oral prednisone was used for a median time of 12 months (range: 9-22 months). A daily prednisone dose $\leq 10 \mathrm{mg}$ was achieved in all 15 cases within a median of 8 months (range: 4-17 months). Four patients $(26.7 \%)$ had to switch from azathioprine to mycophenolate mofetil due to refractoriness ( 2 cases) and intolerance (gastrointestinal symptoms and pharmacodermia, one case each). The median interval to this switch was 223.5 days (range: 124-252 days). All patients were still under IMT after 24 months.

During the follow-up, 5 patients ( 9 eyes, $30 \%$ ) had anterior uveitis flare and/or macular edema. All these patients had inflammation controlled with IMT increment, except one patient (2 eyes, 6.7\%) who had refractory anterior uveitis flare with CNV at month 18 of follow-up in spite of IMT increment. All eyes persisted with subclinical inflammation during the follow-up: DD were still present in all eyes at M24 and DD score fluctuation with worsening was present in 24 eyes (80\%), CT increase was detected in 15 eyes (50\%) and perivascular leakage on FA was present in 17 eyes (56.7\%). Choroidal folds were observed in the acute phase in five eyes (16.7\%). An illustrative case is demonstrated in Figure 1.

Regarding ocular complications, during the follow-up, cataract was observed in seven eyes (23.3\%); ocular hypertension in 14 eyes (46.7\%); subretinal fibrosis in 11 eyes (36.7\%); and, CNV in four eyes of 2 patients (13.3\%). One patient with CNV was under azathioprine and another patient under mycophenolate mofetil therapy at the time of CNV diagnosis. At the 24-month evaluation, SGF was observed in 18 eyes $(60 \%)$. No patients had cataract extraction or other ocular surgeries during the follow-up.

Concerning ffERG evaluation, all parameters improved within 12 months of follow-up but remained subnormal ffERG in 23 eyes (76.7\%) at the M24 follow-up point. Comparing ffERG parameters of M12 and M24 points, they remained stable or improved in 20 eyes $(66.7 \%)$ and they worsened in at least one parameter in 10 eyes (33.3\%). (Table 2 and Supplemental Table 2)

Some correlations between clinical and functional data were observed. Among the 18 eyes with SGF, 17 eyes $(94.4 \%)$ had subnormal ffERG at the M24 evaluation $(p=0.022)$. Fibrosis was more frequently observed in eyes that had anterior uveitis flare $(p=0.007)$. Higher number of cells in CSF was observed in the worsening ffERG group than in the stable ffERG group (median 262.5 cells versus 32 cells, respectively, $\mathrm{p}=0.011)$. The presence of anterior uveitis flare and/or macular edema was associated with a higher FA score at M12 $(p=0.022)$ and M24 $(p=0.002)$ and a higher DD score at M6 $(p=0.002)$.

Evaluating the impact of very early treatment (CS $<14$ days and therapeutic dose IMT $<4$ months), it was observed that an earlier treatment was related with a lower ICGA score at M1 $(p=0.012)$ and a lower DD score at M1 ( $p=0.047)$. Besides that, an earlier treatment was related to a faster period to reach CSsparing effect $(p=0.018)$. (Table 3$)$

\section{Discussion}

In this prospective study, 15 patients with acute onset VKHD were treated with early AZA in association with high-dose corticosteroid. Acute inflammatory signs rapidly receded with BCVA recovery of $\geq 20 / 40$ in $90 \%$ of eyes at one month of follow-up and, at final follow-up, all eyes had BCVA $\geq 20 / 25$. Chronic 
refractory anterior uveitis was observed in one patient (2 eyes, 6.7\%) in spite of IMT increment. ffERG parameters remained stable in $66.7 \%$ of the eyes despite persistence of subclinical signs of inflammation in all included eyes.

Immunosuppression therapy as first-line treatment in acute VKHD has been proposed to achieve faster control of the uveitis and to facilitate earlier tapering of the corticosteroids(18). However, there are very few studies that systematically evaluated visual and inflammatory outcomes in acute VKHD with early IMT. In 2012, Abu El-Asrar et al. described the effectiveness of early IMT in acute VKHD(33). These authors used mycophenolate mofetil with high-dose corticosteroid as a first-line therapy in 19 acute VKHD patients and compared their results with a historical group of 68 acute VKHD patients treated with steroid monotherapy with a mean follow-up of 27 months (range, 16-54 months)(33). The early IMT group had better results than the CS group: visual acuity of $20 / 20$ (74\% versus $38 \%$ ); recurrent inflammation (3\% versus $18 \%$ ); complications such as SGF (0\% versus $100 \%)(33)$. In 2017 , the same group confirmed their results by evaluating the long-term effectiveness (mean follow-up period of 37 months; range, 9-120 months) of mycophenolate mofetil ( $2 \mathrm{~g} /$ day) as a first-line therapy associated with CS in 38 prospectively followed patients $(33,34)$. Other authors did not present such a clear-cut difference between first-line IMT with CS and CS alone or with late IMT. Chee et al, in a retrospective study, compared outcomes of patients with IMT before 6 weeks $(n=15)$ and after 6 weeks of disease onset $(n=14)$ and did not find a difference in SGF prevalence and in the proportion of eyes with resolved or chronic recurrent uveitis(19). Visual acuity was significantly better in the early IMT patients at the $4^{\text {th }}$ year of follow-up. Chee et al. also compared data of patients with IMT within 3 months from disease onset ( $\mathrm{n}=29$ patients) and patients with CS only or with late IMT ( $\mathrm{n}=60$ patients) and did not find differences in SGF prevalence; the proportion of eyes with resolved ( $41.4 \%$ versus $33.3 \%)$, with chronic $(51.7 \%$ versus $28.3 \%)$ or chronic recurrent $(6.9 \%$ versus $38.4 \%)$ indicated significantly more eyes became chronic but fewer chronic recurrent among those with IMT within 3 months. These authors also found significantly better visual acuity in patients with IMT within 3 months at the $3^{\text {rd }}$ year of follow-up(19). Recently a prospective, multicenter, randomized and non-inferiority trial was conducted in Japan comparing the efficacy and safety of a combination therapy of prednisolone and cyclosporine ( $\mathrm{n}=34$ patients) and corticosteroid pulse therapy ( $n=36$ patients) in VKHD with a 1 year follow-up. These authors observed lower recurrence/worsening risk, sunset glow fundus grade, and cataract rate in the combination group than in the corticosteroid group (35).

The following considerations could be made, when comparing our data with those previous studies.

Firstly, the timing of introduction of IMT may vary from a very early treatment, defined as within 2 weeks from disease onset(36) to an interval up to 6 months $(19,35,37,38)$. The rapid control of acute inflammatory signs depends primarily on the early use of high-dose systemic corticosteroid followed by its slow tapering(37). Conventional IMT takes at least 2 to 3 months to reach its ideal therapeutic action(17); therefore, the IMT introduction interval should not impact significantly on the outcomes. In our study, 13 patients (86.7\%) achieved the AZA therapeutic dose within 4 months. Indeed, in the present study the comparison between patients treated with very early treatment (CS within 14 days and 
therapeutic dose of AZA within 4 months) and those patients treated otherwise (CS>14 days and/or therapeutic dose of AZA after 4 months) pointed out that very early group had a lower DD score at M1 than the other group and reached CSE in a shorter median time. We did not find differences in other outcomes including inflammatory or functional parameters. In the literature, early IMT has already shown its benefits on $\operatorname{CSE}(33,34,37,40)$.

Secondly, besides azathioprine and mycophenolate mofetil, other drugs have been used, e.g., methotrexate, cyclosporine, cyclophosphamide and biologics, with slight differences in CSE and frequency of ocular complications(18, 37, 40-45). The most frequent IMT used in VKHD patients by Chee et al. was azathioprine, followed by methotrexate and mycophenolate mofetil(19). Concerning the differences in antimetabolites prescribed, azathioprine is approved by the Public Brazilian Healthcare System to be used in non-infectious uveitis, while mycophenolate mofetil is not. Both immunosuppressive drugs are effective in controlling non-infectious uveitis $(17,40,44)$.

Thirdly, study designs differ. The unique characteristics of the present study are as follows: prospective with all included patients with the same 24-month follow-up period; clinical, multimodal and electroretinogram exams at predefined intervals and treatment management based on clinical inflammation and on electroretinogram parameters. On the other hand, most studies analyzed, as main outcomes, visual acuity, recurrences based on clinical signs and ocular complications. Few studies included systematic multimodal imaging, but the results are not described $(33,34,37,38)$. An exception is Chee's study in which choroidal thickness decreased over time and average ICGA score decreased throughout the follow-up period and, at the 5-year follow-up, 2 patients had persistent DD(19). Most studies had varied follow-up periods after the minimum initial six months.

Concerning subclinical signs in the non-acute phase of VKHD, they may indicate choroidal inflammation; however, in our study, even after 24-month of follow-up, we could observe that they did not impact in visual function when evaluated by VA, and, in at least two thirds of our patients, when evaluated by ffERG. It should be noted that subclinical signs, more importantly those observed on ICGA, are difficult to quantify and interpret. Herbort et al. evaluated all ICGA and FA signs and their fluctuation(24), while Chee et al. only evaluated the ICGA signs as a categorical item(19). In the present study, they were systematically evaluated but their isolated presence was not used to indicate systemic treatment increment; retinal function measurements were carried out and worsening of ffERG parameters then directed treatment change or not. Sakata et al. had previously demonstrated that choroidal subclinical inflammation fluctuated along the course of the disease, i.e. worsened and improved without treatment change(13). Souto et al. demonstrated that this subclinical choroiditis improved along the course of the disease even after systemic treatment is discontinued(46) and, further, Souto et al. also demonstrated that this subclinical choroiditis did not impact on patients' quality of life(47). On the other hand, Herbort et al. strongly suggest that monitorization of VKHD through ICGA is essential to avoid chronicity and sunset glow fundus(36). Therefore, subclinical signs may be important to a global understanding of the inflammatory status of the disease but, if presented isolated, may be not enough to dictate treatment intervention. Nevertheless, our study pointed out that a portion of the $33 \%$ of patients with worsening ERG 
could deserve a more aggressive treatment. Thus, biomarkers of disease severity and the understanding of timing for being more aggressive should be pursued. Our study is the first prospective study with systematic multimodal and ffERG evaluation and early IMT; therefore, further studies are compelling to still clarify these points.

Despite the prospective study design with a systematic follow-up, some limitations should be considered. Firstly, a larger cohort sample size and a longer follow-up could add statistical power to detect differences. Secondly, ffERG was used as an objective method to evaluate retinal function and ffERG parameters were defined to compare groups. Nevertheless, the definition of retinal dysfunction progression was based on other inflammatory and non-inflammatory retinal/choroidal diseases(2729) due to very scarce literature using ERG in VKHD. Furthermore, ffERG is a complex exam, which is not always available and the definition of worsening or not should be validated in future studies (Supplemental Table 2). Thus, besides ffERG, other means of evaluating the impact of inflammation on visual function may play a role in VKHD appraisal, e.g., visual field, microperimetry, contrast sensibility and self-reported quality of life questionnaires(47-49). It is important to highlight that all of these methods are subjective and their interpretation should be done cautiously. Thirdly, no control group using late IMT was presented to compare with the early IMT group. Nevertheless, our group recently published a study with a similar prospective design using corticosteroid only or late IMT in acute VKHD(13). Lastly, even though patients were recruited from early disease onset, there is heterogeneity in the interval to diagnosis and in disease severity.

In conclusion, early azathioprine with high-dose corticosteroid was effective in improving BCVA with control of clinical inflammation in more than $90 \%$ of patients. Isolate subclinical choroidal inflammation persistence may not be enough to indicate treatment increment, since VA was $20 / 25$ in $100 \%$ of patients and visual function evaluated by ffERG remained stable in two thirds of patients, even after 24 months of follow-up. However, selected patients, particularly those with worsening ffERG, may deserve further treatment.

\section{Declarations}

\section{Ethics approval}

All procedures performed in this study were in accordance with the ethical standards of the institutional research committee (CAPPesq 0496/11) and the 1964 Helsinki declaration and its later amendments (clinical trials NCT03399175).

\section{Consent to participate}

Informed consent was obtained from all individual participants included in the study.

\section{Consent for publication}

Not applicable. 
Availability of data and material

The authors confirm that the data supporting the findings of this study are available within the article and/or its supplementary materials.

\section{Code availability}

The authors declare that data analysis and statistical tests were conducted using SPSS20.0 (SPSS Science, Chicago, IL, USA).

\section{Competing interests}

The authors declare that they have no conflict of interest.

\section{Funding}

This study was supported by the São Paulo Research Foundation (FAPESP): 2011/19194-4 (VMS) and 2011/50936-7 (JHY). It was also supported by CNPq-Conselho Nacional de Desenvolvimento Científico e Tecnológico (310422/2018-3 - JHY). The funding organizations had no role in the design or conduct of this research.

\section{Authors' contributions}

All authors contributed to the manuscript as follows:

MML, VMS, FMSS, RFBGM, PFCN, MKO, CEH, JHY: conception, design, analysis, and interpretation of data.

MML, JHY: drafting the paper.

MKO, CEH, JHY: revising paper critically for important intellectual content.

MML, VMS, FMSS, RFBGM, PFCN, MKO, CEH, JHY: final approval of version sent out for publishing.

\section{Acknowledgments}

The authors thank Rogério Ruscitto Prado, PhD in Statistics, Department of Public Health and Preventive Medicine, University of São Paulo School of Medicine, São Paulo, Brazil, for his assistance in statistical analysis; Smairah Frutuoso Abdallah (SFA) and Cintia Kanenobu, Ophthalmic technicians, Department of Ophthalmology, Hospital das Clínicas HCFMUSP, Faculdade de Medicina, Universidade de São Paulo, São Paulo, Brazil, for their availability and contribution to performing most of the ophthalmologic exams on our patients and Maria Lucia C Marin, Biomedical Scientist, and Dr Helcio Rodrigues, Biologist, Immunology Lab, Histocompatibility Group, InCor - Heart Institute, University of São Paulo, São Paulo, Brazil, for HLA typification. 


\section{References}

1. Moorthy RS, Inomata H, Rao NA (1995) Vogt-Koyanagi-Harada syndrome. Surv Ophthalmol 39:265292.

2. Damico FM, Bezerra FT, Silva GC, Gasparin F, Yamamoto JH (2009) New insights into Vogt-KoyanagiHarada disease. Arq Bras Oftalmol 72:413-420.

3. Lavezzo MM, Sakata VM, Morita C, Rodriguez EE, Abdallah SF, da Silva FT, et al (2016) VogtKoyanagi-Harada disease: review of a rare autoimmune disease targeting antigens of melanocytes. Orphanet J Rare Dis 11:29. doi: 10.1186/s13023-016-0412-4

4. Herbort CP, Jr., Abu El Asrar AM, Yamamoto JH, Pavesio CE, Gupta V, Khairallah M, et al (2017) Reappraisal of the management of Vogt-Koyanagi-Harada disease: sunset glow fundus is no more a fatality. Int Ophthalmol 37(6):1383-1395.

5. Du L, Kijlstra A, Yang P (2016) Vogt-Koyanagi-Harada disease: Novel insights into pathophysiology, diagnosis and treatment. Prog Retin Eye Res 52:84-111.

6. Gouveia EB, Yamamoto JH, Abdalla M, Hirata CE, Kubo P, Olivalves E (2004) Causas das uveítes em serviço terciário em São Paulo, Brasil. Arq Bras Oftalmol 67:139-145.

7. Belfort Jr R, Nishi M, Hayashi S, Abreu MT, Petrilli AM, Plut RC (1988) Vogt-Koyanagi-Harada's disease in Brazil. Jpn J Ophthalmol 32:344-347.

8. Gonzalez Fernandez D, Nascimento H, Nascimento C, Muccioli C, Belfort R, Jr (2017) Uveitis in Sao Paulo, Brazil: 1053 New Patients in 15 Months. Ocul Immunol Inflamm 25:382-387.

9. Jones NP (2015) The Manchester Uveitis Clinic: the first 3000 patients--epidemiology and casemix. Ocul Immunol Inflamm 23:118-126.

10. Snyder DA, Tessler HH (1980) Vogt-Koyanagi-Harada syndrome. Am J Ophthalmol 90:69-75.

11. Ohguro N, Sonoda KH, Takeuchi M, Matsumura M, Mochizuki M (2012) The 2009 prospective multicenter epidemiologic survey of uveitis in Japan. Jpn J Ophthalmol. 2012;56(5):432-5.

12. Yang P, Zhang Z, Zhou H, Li B, Huang X, Gao Y, et al (2005) Clinical patterns and characteristics of uveitis in a tertiary center for uveitis in China. Curr Eye Res 30:943-948.

13. Sakata VM, Lavezzo MM, da Silva FT, Rodriguez EEC, Morita C, Abdallah SF, et al (2019) Full-field electroretinogram behavior in Vogt-Koyanagi-Harada disease: a 24-month longitudinal study in patients from acute onset evaluated with multimodal analysis. Graefes Arch Clin Exp Ophthalmol 227: 2285-2295.

14. Nakayama M, Keino $H$, Watanabe $T$, Okada AA (2019) Clinical features and visual outcomes of 111 patients with new-onset acute Vogt-Koyanagi-Harada disease treated with pulse intravenous corticosteroids. Br J Ophthalmol. 103:274-278.

15. Urzua CA, Chen P, Chaigne-Delalande B, Liu B, Anguita R, Guerrero J, et al (2019) Glucocorticoid receptor alpha and MKP-1 as candidate biomarkers for treatment response and disease activity in Vogt-Koyanagi-Harada disease. Am J Ophthalmol 207:319-325 . 
16. Herbort CP, Jr., Tugal-Tutkun I, Khairallah M, Abu El Asrar AM, Pavésio CE, Soheilian M (2020) VogtKoyanagi-Harada disease: recurrence rates after initial-onset disease differ according to treatment modality and geographic area. Int Ophthalmol 40:2423-2433.

17. Jabs DA, Rosenbaum JT, Foster CS, Holland GN, Jaffe GJ, Louie JS, et al (2000) Guidelines for the use of immunosuppressive drugs in patients with ocular inflammatory disorders: recommendations of an expert panel. Am J Ophthalmol 130:492-513.

18. Paredes I, Ahmed M, Foster CS (2006) Immunomodulatory therapy for Vogt-Koyanagi-Harada patients as first-line therapy. Ocul Immunol Inflamm. 14:87-90.

19. Ei Ei Lin O, Chee SP, Wong KKY, Hla Myint H. Vogt-Koyanagi-Harada Disease Managed With Immunomodulatory Therapy Within 3 Months of Disease Onset. Am J Ophthalmol. 2020;220:37-44.

20. Papasavvas I, Tugal-Tutkun I, Herbort CP, Jr (2020) Vogt-Koyanagi-Harada is a Curable Autoimmune Disease: Early Diagnosis and Immediate Dual Steroidal and Non-Steroidal Immunosuppression are Crucial Prerequisites. J Curr Ophthalmol 32:310-314.

21. Bouchenaki N, Herbort CP (2001) The contribution of indocyanine green angiography to the appraisal and management of Vogt-Koyanagi-Harada disease. Ophthalmology 108:54-64.

22. Read RW, Holland GN, Rao NA, Tabbara KF, Ohno S, Arellanes-Garcia L, et al (2001) Revised diagnostic criteria for Vogt-Koyanagi-Harada disease: report of an international committee on nomenclature. Am J Ophthalmol. 131:647-652.

23. da Silva FT, Hirata CE, Olivalves E, Oyamada MK, Yamamoto JH (2009) Fundus-based and electroretinographic strategies for stratification of late-stage Vogt-Koyanagi-Harada disease patients. Am J Ophthalmol 148:939-945 e3.

24. Tugal-Tutkun I, Herbort CP, Khairallah M, Angiography Scoring for Uveitis Working G (2010) Scoring of dual fluorescein and ICG inflammatory angiographic signs for the grading of posterior segment inflammation (dual fluorescein and ICG angiographic scoring system for uveitis). Int Ophthalmol 30:539-552.

25. Herbort CP, Mantovani A, Bouchenaki N (2007) Indocyanine green angiography in Vogt-KoyanagiHarada disease: angiographic signs and utility in patient follow-up. Int Ophthalmol 27:173-182.

26. McCulloch DL, Marmor MF, Brigell MG, Hamilton R, Holder GE, Tzekov R, et al (2015). ISCEV Standard for full-field clinical electroretinography (2015 update). Documenta ophthalmologica Advances in ophthalmology 130:1-12.

27. Berson EL, Sandberg MA, Rosner B, Birch DG, Hanson AH (1985). Natural course of retinitis pigmentosa over a three-year interval. Am J Ophthalmol 99:240-251.

28. Priem HA, Oosterhuis JA (1988) Birdshot chorioretinopathy: clinical characteristics and evolution. $\mathrm{Br}$ J Ophthalmol 72:646-659.

29. Hirose T, Katsumi O, Pruett RC, Sakaue H, Mehta M (1991) Retinal function in birdshot retinochoroidopathy. Acta Ophthalmol (Copenh) 69:327-337.

30. Berson EL, Gouras P, Hoff M (1969) Temporal aspects of the electroretinogram. Arch Ophthalmol 81:207-214. 
31. Jabs DA, Nussenblatt RB, Rosenbaum JT, Standardization of Uveitis Nomenclature Working G (2005) Standardization of uveitis nomenclature for reporting clinical data. Results of the First International Workshop. Am J Ophthalmol 140:509-516.

32. Sakata VM, Morita C, Lavezzo MM, Rodriguez EEC, Abdallah SF, Pimentel SLG, et al (2021) Outcomes of Intravitreal Bevacizumab in Choroidal Neovascularization in Vogt-Koyanagi-Harada Disease - A Prospective Study. Ocul Immunol Inflamm 29:572-578.

33. Abu El-Asrar AM, Hemachandran S, Al-Mezaine HS, Kangave D, Al-Muammar AM (2012) The outcomes of mycophenolate mofetil therapy combined with systemic corticosteroids in acute uveitis associated with Vogt-Koyanagi-Harada disease. Acta ophthalmologica 90:e603-8.

34. Abu El-Asrar AM, Dosari M, Hemachandran S, Gikandi PW, Al-Muammar A (2017) Mycophenolate mofetil combined with systemic corticosteroids prevents progression to chronic recurrent inflammation and development of 'sunset glow fundus' in initial-onset acute uveitis associated with Vogt-Koyanagi-Harada disease. Acta ophthalmologica 95:85-90.

35. Ono T, Goto H, Sakai T, Nitta F, Mizuki N, Takase H, et al (2021) Comparison of combination therapy of prednisolone and cyclosporine with corticosteroid pulse therapy in Vogt-Koyanagi-Harada disease. Jpn J Ophthalmol doi: 10.1007/s10384-021-00878-w

36. Herbort CP, Jr., Abu El Asrar AM, Takeuchi M, Pavesio CE, Couto C, Hedayatfar A, et al (2019) Catching the therapeutic window of opportunity in early initial-onset Vogt-Koyanagi-Harada uveitis can cure the disease. Int Ophthalmol 39:1419-1425.

37. Urzua CA, Velasquez V, Sabat P, Berger O, Ramirez S, Goecke A, et al (2015) Earlier immunomodulatory treatment is associated with better visual outcomes in a subset of patients with Vogt-Koyanagi-Harada disease. Acta ophthalmologica 93:e475-480.

38. Yang P, Ye Z, Du L, Zhou Q, Qi J, Liang L, et al (2018) Novel treatment regimen of Vogt-KoyanagiHarada disease with a reduced dose of corticosteroids combined with immunosuppressive agents. Curr Eye Res 43:254-261.

39. Lai TY, Chan RP, Chan CK, Lam DS (2009) Effects of the duration of initial oral corticosteroid treatment on the recurrence of inflammation in Vogt-Koyanagi-Harada disease. Eye (Lond) 23:543548.

40. Shen E, Rathinam SR, Babu M, Kanakath A, Thundikandy R, Lee SM, et al (2016) Outcomes of VogtKoyanagi-Harada Disease: A Subanalysis From a Randomized Clinical Trial of Antimetabolite Therapies. Am J Ophthalmol 168:279-286.

41. Kim SJ, Yu HG (2007) The use of low-dose azathioprine in patients with Vogt-Koyanagi-Harada disease. Ocul Immunol Inflamm 15:381-387.

42. Couto C, Schlaen A, Frick M, Khoury M, Lopez M, Hurtado E, et al (2018) Adalimumab Treatment in Patients with Vogt-Koyanagi-Harada Disease. Ocul Immunol Inflamm 26:485-489.

43. Cuchacovich M, Solanes F, Diaz G, Cermenati T, Avila S, Verdaguer J, et al (2010) Comparison of the clinical efficacy of two different immunosuppressive regimens in patients with chronic vogtkoyanagi-harada disease. Ocul Immunol Inflamm 18:200-207. 
44. Arcinue CA, Radwan A, Lebanan MO, Foster CS (2013) Comparison of two different combination immunosuppressive therapies in the treatment of Vogt-Koyonagi-Harada syndrome. Ocul Immunol Inflamm 21:47-52.

45. Yang P, Ren Y, Li B, Fang W, Meng Q, Kijlstra A (2007) Clinical characteristics of Vogt-KoyanagiHarada syndrome in Chinese patients. Ophthalmology 114:606-614.

46. Silveira Souto FM, Missaka RFBG, Lavezzo MM, Nóbrega PFC, Sakata VM, Oyamada MK, et al (2021) Vogt-Koyanagi-Harada disease (VKHD) after systemic treatment discontinuation. Investigative Ophthalmology \& Visual Science 62:1405.

47. Souto FMS, Missaka R, Magalhaes BM, Caetano VMC, Takiuti JT, Lavezzo MM, et al (2021) Impact of Inflammation and Treatment on Self-reported Quality of Life in Patients with Non-acute VogtKoyanagi-Harada Disease (VKHD). Ocul Immunol Inflamm 29:137-148.

48. Yang P, Sun M, Liu X, Zhou H, Fang W, Wang L, et al (2012). Alterations of color vision and central visual field in patients with Vogt-Koyanagi-Harada syndrome. J Ophthalmic Inflamm Infect. 2:75-79.

49. Abu El-Asrar AM, Al-Mezaine HS, Hemachandran S, Hariz R, Kangave D (2012) Retinal functional changes measured by microperimetry after immunosuppressive therapy in patients with VogtKoyanagi-Harada disease. Eur J Ophthalmol 22:368-375.

\section{Tables}

Table 1-Demographics, clinical and subclinical signs of inflammation, and ocular complications of 15 patients with Vogt-Koyanagi-Harada disease at baseline (early acute phase) and during the 24month follow-up 


\section{Description}

Female (\%)

14

(93.3)

Age, median (range)

$32 \quad(19-46)$

Race, patients (\%)

White

$6 \quad(40)$

Pardo

9

HLA-DRB1*0405, patients (\%)

5

Interval between symptoms and treatment, patients (\%)

0-14 days

5

14-30 days

7

$>30$ days

3

Interval between CS therapy and azathioprine introduction, patients (\%)

Concomitant

Within one month

From one to 4 months

Best corrected visual acuity, LogMAR, median (range)

Baseline

M1

M12

M24

Subfoveal choroidal thickness, $\mu \mathrm{m}$, median (range)

Baseline $^{a}$

M1

M12

M24

FA score ${ }^{\mathrm{b}}$, median (range)

Baseline

M1
M12

Baseline
$1 \quad(6.7)$

$5 \quad$ (33.3)

$9 \quad(60)$

$0.7 \quad(0.0-2.0)$

$0.075(0.0-2.0)$

$0.0 \quad(0.0-0.7)$

$0.0 \quad(0.0-0.15)$

$774 \quad(454-1167)$

529.5 (310-940)

346.5 (122-667)

$310 \quad(139-536)$
$6.5(2-11)$

$2(0-4)$

$1(0-3)$ 
ICGA score $^{\mathrm{b}}$, median (range)

Baseline

M1

M12

M24
$11.5(6-17)$

$8 \quad(5-13)$

$5 \quad(4-8)$

$4.5 \quad(3-5)$

Dark dots score, median (range)

Baseline

M1

M12

M24

Inflammatory signs during follow-up, eyes (\%)

Anterior chamber cells (recurrent anterior uveitis)

Macular edema

Perivascular leakage

Choroidal thickness fluctuation ${ }^{c}$

Dark dots score fluctuation ${ }^{d}$

Complications, eyes (\%)

Cataract

Ocular hypertension / glaucoma

Sunset glow fundus

Subretinal fibrosis

Choroidal neovascularization

Fundus classification at $\mathrm{M} 24^{\mathrm{e}}$, eyes (\%)

Mild

Moderate

Severe

Full-field electroretinogram, eyes (\%)
$8 \quad(6-8)$

$8 \quad(4.5-8)$

$5 \quad(4-8)$

$4.5 \quad(3-5)$


Stable

Worsening

10

Treatment, patients (\%)

Single immunosuppressant drug 11

Switch of immunosuppressant drug due to refractoriness / intolerance

4

a Due to technical issues (e.g. considerable retinal detachment not fitting into scan), some measures of subfoveal choroidal thickness were greater than the presented measure; ${ }^{b}$ Score based on Tugal-Tutkun et al., 2010); 'Increase of $30 \%$ or more of choroidal thickness among three different consecutive points in time; ${ }^{d}$ Increase in the number of DD by region, whether in the posterior pole or in each quadrant, followed by a diminishment. Objectively was defined as a variation in the final score among three different points in time $\geq 0.5$ (based on and modified from Tugal-Tutkun et al., 2010); ${ }^{\text {e } B a s e d ~ o n ~ f u n d o s c o p i c ~}$ classification described by da Silva et al., 2009.

Table 2 - Full-field electroretinogram results at baseline, 12 and 24 months (median value) in 15 patients with Vogt-Koyanagi-Harada disease followed from acute onset and 30 normal controls 
Parameters $^{a}$

Controls Patients ( $n=30$ eyes)

$(n=60$

eyes)

M1

M12

Scotopic (ms) b wave implicit time

amplitude $(\mu \mathrm{V})$

93.2

(81.9-106)

71.5

(1.3-343)
96.3

(85.1-112.4)

209.9

(111.8-

286.2)
M24

92.7

$<0.001$

(79.5-108.3)

194.8

0.149

(88.5-261.9)

Maximum scotopic

(ms)

a wave implicit time

22

24.7

23.7

23.2

$<0.001$

(18-23)

(22.3-28.2)

(21.7-24.7)

(21.4-25)

amplitude $(\mu \mathrm{V}) \quad 299.5$

170.5

223.9

223

$<0.001$

(211-440)

(43.2-419)

(89.4-372.3)

(85.4-325.1)

(ms)

b wave implicit time

44

54.2

50.8

49.8

$<0.001$

amplitude $(\mu \mathrm{V})$

(36-50)

(48.7-61.9)

(46.7-58.7)

(46.4-55.8)

(348-789)

311.4

502.3

503.4

0.364

(156-798)

(313-790.1)

(276.2-

616.9)

Oscillatory potential $(\mu \mathrm{V})$

122.2

70.4

126.2

(58.3-288.4)

121.1

$>0.999$

(92.3-

227.9)

(23.5-

302.1)

\section{Photopic} (ms)

a wave implicit time

15

17.2

16.4

16.3

$<0.001$

(14-17)

(15.3-20.3)

(14.1-17.6)

(14.4-17.9)

amplitude $(\mu \mathrm{V}) \quad 49.2$

33.2

39.7

40.5

0.002

(33.6-89.5)

(14.7-67.7)

(21.1-58.5)

(13.1-68.7)

(ms)

b wave implicit time

31

33.8

31.4

31.7

0.457

(27-34)

(28.5-39.6)

(29.1-34.9)

(28.2-33.8)

amplitude $(\mu \mathrm{V}) \quad 224.5$

147.5

158.9

163.8

0.008

(106-367)

(28.3-315)

(77.9-300)

(58-298.5) 


\section{Flicker $30 \mathrm{~Hz}$}

67.3

36.1

44.9

46

0.001

$(41.9-122.1)$

(14-102)

$(21.8-86.6)$

$(12.2-90.3)$

ERG: electroretinogram; ${ }^{\mathrm{a} E R G}$ measures represented as median (minimum-maximum); ${ }^{\mathrm{b}} p$ value comparing controls $x$ patients at 24-month follow-up-generalized estimated equations with normal distribution and identity link function, supposing an exchangeable correlation matrix between the eye and moments followed by Bonferroni test for multiple comparisons

Table 3 -Early treatment (corticosteroid initiated $\leq 14$ days and therapeutic dose of non-steroidal immunosuppressive drug therapeutical dose within 4 months versus corticosteroid initiated $>14$ days and/or non-steroidal immunosuppressive drug therapeutical dose after 4 months) groups of patients with Vogt-Koyanagi-Harada disease followed from acute onset with their characteristics 


\begin{tabular}{|c|c|c|c|c|c|}
\hline & Treatm & & & & \\
\hline & $\begin{aligned} C S & <1 \\
\mathrm{IMT}<4 \mathrm{n} & \end{aligned}$ & and & $\begin{array}{r}C S>1 \\
I M T>4\end{array}$ & and/or & $p$ \\
\hline N patients (eyes) & 6 & & 9 & & \\
\hline Age, years, median (range) & $\begin{array}{l}33.5 \\
37)\end{array}$ & $(26-$ & $\begin{array}{l}30 \\
46)\end{array}$ & $(19-$ & $0.955^{\star}$ \\
\hline Pleocitosis, cells $/ \mathrm{mm}^{3}$, median (range) & $\begin{array}{l}180 \\
285)\end{array}$ & $(2-$ & $\begin{array}{l}130 \\
520)\end{array}$ & (3- & $>0.999^{*}$ \\
\hline Best corrected visual acuity (logMAR), $n$ & & & & & \\
\hline baseline & 0.85 & $(0-2)$ & 0.26 & $(0-2)$ & $0.840^{\#}$ \\
\hline at M1 & $\begin{array}{l}0.225 \\
0.5)\end{array}$ & $(0-$ & 0 & $(0-2)$ & $0.986^{\#}$ \\
\hline at M12 & 0 & $(0-0)$ & $\begin{array}{l}0 \\
0.7)\end{array}$ & $(0-$ & \& \\
\hline at M24 & $\begin{array}{l}0 \\
0.04)\end{array}$ & $(0-$ & $\begin{array}{l}0 \\
0.15)\end{array}$ & $(0-$ & $0.573^{\#}$ \\
\hline Subfoveal choroidal thickness, $\mu \mathrm{m}$, mec & & & & & \\
\hline baseline ${ }^{a}$ & $\begin{array}{l}712.5 \\
(521-11\end{array}$ & & $\begin{array}{l}826 \\
1113)\end{array}$ & $(454-$ & $0.611^{\#}$ \\
\hline at $\mathrm{M} 1$ & $\begin{array}{l}511 \\
(310-79\end{array}$ & & $\begin{array}{l}536.5 \\
940)\end{array}$ & $(346-$ & $0.314^{\#}$ \\
\hline at M12 & $\begin{array}{l}305 \\
(122-46\end{array}$ & & $\begin{array}{l}372.5 \\
667)\end{array}$ & $(269-$ & $0.056^{\#}$ \\
\hline at M24 & $\begin{array}{l}279 \\
(139-50\end{array}$ & & $\begin{array}{l}334.5 \\
536)\end{array}$ & $(259-$ & $0.104^{\#}$ \\
\hline FA score, ${ }^{\mathrm{b}}$ median (range) & & & & & \\
\hline Baseline & $\begin{array}{l}7.5 \\
10)\end{array}$ & $(5-$ & 6.5 & $(2-11)$ & $0.482^{\#}$ \\
\hline at M1 & 2 & $(0-3)$ & 2 & $(2-4)$ & $0.108^{\#}$ \\
\hline at M12 & 1.5 & $(0-3)$ & 1 & $(0-3)$ & $0.717^{\#}$ \\
\hline at M24 & 0.5 & $(0-2)$ & 1 & $(0-3)$ & $0.643^{\#}$ \\
\hline ICGA score, ${ }^{\mathrm{b}}$ median (range) & & & & & \\
\hline Baseline & $\begin{array}{l}8 \\
17)\end{array}$ & (6- & 14 & $(8-16)$ & $0.471^{\#}$ \\
\hline
\end{tabular}


at $\mathrm{M} 1$

$6.75 \quad(5-\quad 9$

13)

at $\mathrm{M} 12$

5
$7.5)$

at M24

5
$5)$

(3.5- $\quad 4.5$

5.25

(4-8)

$0.787^{\#}$

Dark dots score, ${ }^{\mathbf{c}}$ median (range)

baseline

8

(6-8) 8

(8-8) $\quad 0.202^{\#}$

at $\mathrm{M} 1$

6.25

$(4.5-\quad 8$

$(5-8)$

$0.047^{\#}$

at $\mathrm{M} 12$

5
$7.5) \quad(4-\quad 5$

at M24

$\begin{array}{lllll}5 & (3.5- & 4.5 & (3-5) & 0.415^{\#}\end{array}$

Inflammatory signs, eyes (\%)

Anterior chamber cells (recurrent anterior uveitis) 2

(16.7)

6

(33.3) $0.482^{*}$

Macular edema

1

(8.3) 1

(5.6)

$0.757^{*}$

Perivascular leakage

6

(50)

11

$(61.1)$

$0.665^{\star}$

Choroidal thickness fluctuation ${ }^{d}$

6

(50) 9

(50)

$>0.999^{*}$

Dark dots fluctuation ${ }^{\mathrm{e}}$

$\begin{array}{ll}8 & 16 \\ (66.7) & (88.9)\end{array}$

$0.218^{*}$

Ocular complications, eyes (\%)

Cataract

5

Ocular hypertension / glaucoma

Sunset glow fundus

Subretinal fibrosis

Chorioretinal atrophy

Peripapillary atrophy

Choroidal neovascularization
(41.7)

6

(50)

2

(11.1)

$0.187^{*}$

8

(44.4)

$0.833^{*}$

$5 \quad 13$

(41.7)

(72.2)

$0.215^{*}$

3

(25)

8

(44.4)

$0.397^{*}$

$1 \quad$ (8.3) 8

(44.4)

$0.098^{*}$

6

(16.7)

(33.3)

$0.372^{*}$

2

$0.758^{*}$ 
Fundus classification at M24, ${ }^{\mathrm{f}}$ eyes (\%)

Mild

Moderate

Severe
9

3

0
(75)

(25)

(0) 5
4

(22.2)

(27.8)

Treatment, patients (\%)

$0.103^{@}$

Single immunosuppressant drug

6

(100)

5

Switch of immunosuppressant drug due to refractoriness / intolerance

0

(0) 4

Time to reach CSE (median, months)

7

$(5-8)$

$(4-$

$0.018^{*}$

CS: corticosteroid; IMT: immunosuppressive therapy; CSE: corticosteroid-sparing effect. ${ }^{a}$ Due to technical issues (e.g. considerable retinal detachment not fitting into scan), some measures of subfoveal choroidal thickness were greater than the presented measure; ${ }^{b}$ Score based on Tugal-Tutkun et al., 2010; ${ }^{c}$ Dark

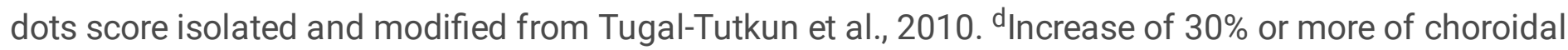
thickness among three different consecutive points in time; ${ }^{\mathrm{e}}$ Increase in the number of DD by region, whether in the posterior pole or in each quadrant, followed by a diminishment. Objectively was defined as a variation in the final score among three different points in time $\geq 0.5$ (based on and modified from Tugal-Tutkun et al., 2010); ${ }^{f}$ Based on fundoscopic classification described by da Silva et al., 2009. *Generalized estimated equations with binomial distribution and logit link function. ${ }^{*}$ Generalized estimated equations with binomial distribution and logit link function, supposing an exchangeable correlation matrix between eyes. @ Fisher's exact test. ${ }^{+}$Generalized estimated equations with Poisson distribution and identity link function. \&: impossible to estimate.

\section{Figures}




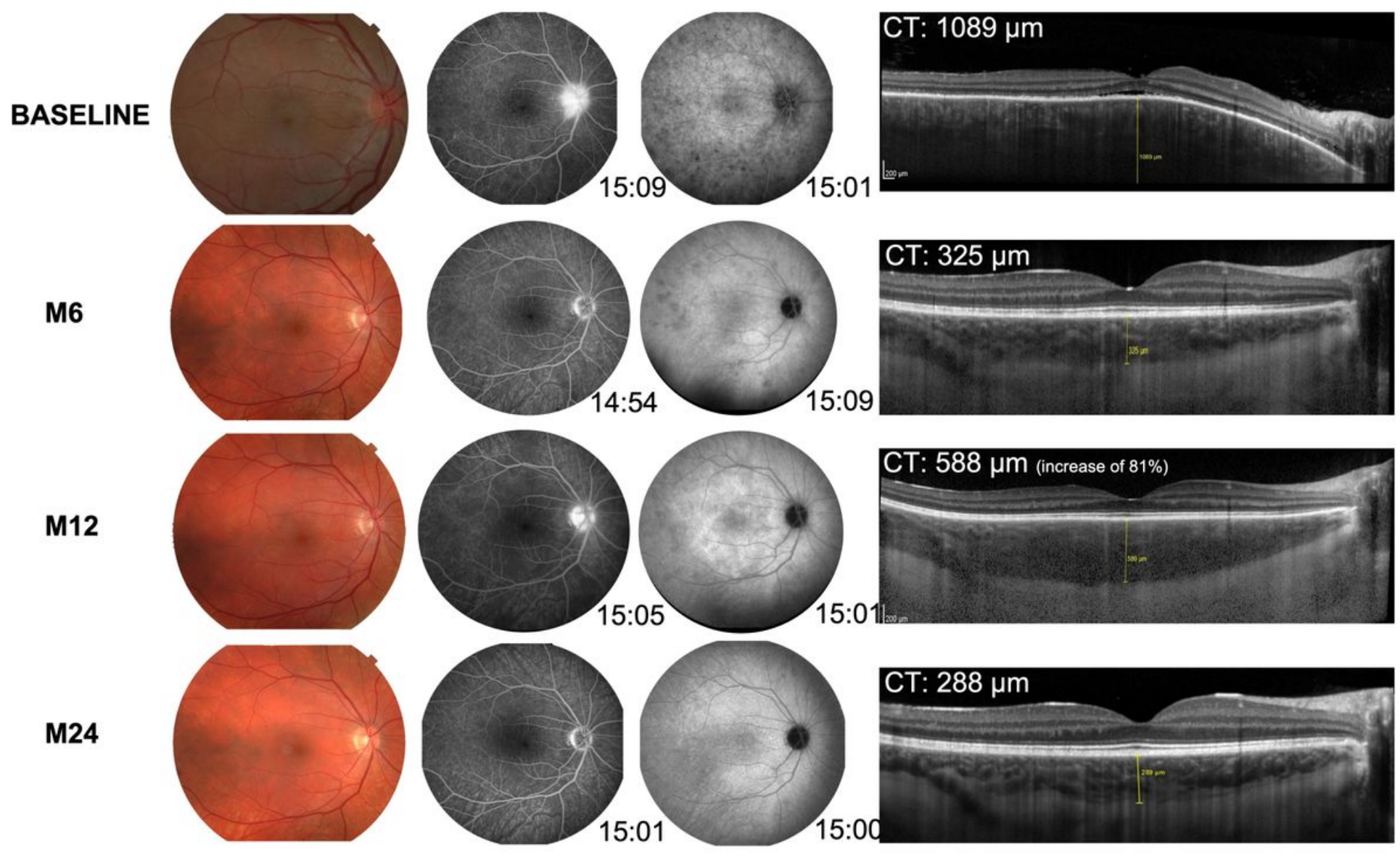

\section{Figure 1}

A patient with Vogt-Koyanagi-Harada disease in both eyes at baseline, M6, M12 and M24 of follow-up. She was included in the stable group based on full-field electroretinogtam scotopic parameters variation between month 12 and month 24. Baseline: Color fundus (CF): presence of optic disc swelling and hyperemia and macular retinal detachment. Fluorescein angiography (FA): presence of pinpoints and optic disc hyperfluorescence. Indocyanine green angiography (ICGA): presence of multiple dark dots and fuzzy vessels. Ocular coherence tomography (OCT): increased choroidal thickness (1089 $\mu \mathrm{m})$. M6: CF: normal fundus appearance. FA: normal FA appearance. ICGA: presence of peripheral dark dots. OCT: decreased choroidal thickness $(325 \mu \mathrm{m})$. M12: CF: normal fundus appearance. FA: presence of optic disc hyperfluorescence. ICGA: presence of peripheral dark dots. OCT: increased choroidal thickness $(588 \mu \mathrm{m})$. M24: CF: presence of mild sunset glow fundus. FA: normal FA appearance. ICGA: presence of very sparse dark dots. OCT: decreased choroidal thickness $(288 \mu \mathrm{m})$.

\section{Supplementary Files}

This is a list of supplementary files associated with this preprint. Click to download.

- SupplementalFigure1VKHD040122.jpg

- SupplementalTable1Steroids080122.docx 
- SupplementalTable2ERG080122.docx

- SupplementalFile.docx 